\title{
New approach for the determination of the characteristics of signals containing fluctuating harmonics
}

\author{
A. Poletaeff* and P. Espel \\ Laboratoire National de Métrologie et d'Essais (LNE), LNE-Trappes ZA de Trappes-Élancourt, 29 avenue Roger Hennequin, \\ 78197 Trappes Cedex, France
}

Received: 14 January 2010 / Accepted: 10 August 2011

\begin{abstract}
This paper describes a new approach for the analysis of signals containing fluctuating harmonics. It is based on the Fourier transform, using windowing to reduce the error arising from analysing the signal over a non integer number of signal periods and an iterative process intended to estimate and correct the errors. Some simulations have been performed to prove the efficiency of the method and showed that the final error arising from the computation generally ranges between a few parts in $10^{10}$ and a few parts in $10^{15}$.
\end{abstract}

Keywords: Harmonics; Fourier analysis; non stationary waveforms

\section{Introduction}

In the next years, Europe will have to face with potential energy shortages as oil and gas supplies run down. At the same time, environment protection policies are increasingly resorting to renewable energy generation and energy management. One consequence will be the diversification of electricity production sources and the need to improve electricity distribution efficiency. In this context, to ensure the quality of the power grid, new quality standards are required and therefore new measurement techniques. Characterisation of complex, non-stationary waveforms is a part of this challenge.

A significant work has already been performed in this domain. Methods aiming to reduce the errors associated with the asynchronous sampling of periodic and "quasi-periodic" signals are widely described in the literature [1-4]. They are particularly useful for direct measurements on the grid. Many papers have also been published on flicker which is a slow and repetitive variation of the voltage and constitute an indicator of the quality degradation of the power grid. One method consists in measuring each minimum and maximum of the modulated signal in the time domain using a high precision digital voltmeter $[5,6]$. The modulation depth can also be determined by using two digital voltmeters (DVMs) and digital logic and software control that ensures that the modulated signals are only measured when either maximum or minimum modulation occurs [7]. A third method is based on spectral analysis using Fourier transform [8]. A comparison of these methods is given in [9]. Another indicator is the presence of harmonics, generally induced when connected appliances present non linear loads. Ac-

${ }^{\star}$ Correspondence: andre.poletaeff@lne.fr curate measurements of such signals have also generated the need for new calibration methods for harmonic analysers. The case of stationary harmonics has already been widely studied $[5,10]$. Characterisation of fluctuating harmonics requires more complex signal analysis methods. Some works have already been performed and are presented in [11-13]. Nevertheless, they only deal with some particular cases, hence the need to develop new methods to expand the application areas. With this problematic in mind, a task which purpose was the development of novel waveform analysis techniques and algorithms has been included in the frame of a European research project on power and energy measurements which is a part of the European Metrology Research Program (EMRP). The present work has been performed at LNE as a partner in this project.

We present here a new approach for the characterization of signals containing fluctuating harmonics based on Fourier analysis. The originality of this approach is the use of an iterative process in order to estimate the error due to applying the Fourier transform in non appropriate conditions (non perfectly periodic signals that do not fulfill the Shannon condition, overlapping between various spectral components) and to correct it. All these error sources are analyzed. Many simulations have been performed to test the method. First simulations illustrate the limitation of the Fourier transform when applied to the analysis of such signals. The other simulations demonstrate the efficiency of the iterative process, give an evaluation of the performance of the method and underscore the influence of the signal parameters.

This method can be applied to the calibration of reference standards that can be used to ensure the traceability of instruments intended for power quality measurements. 


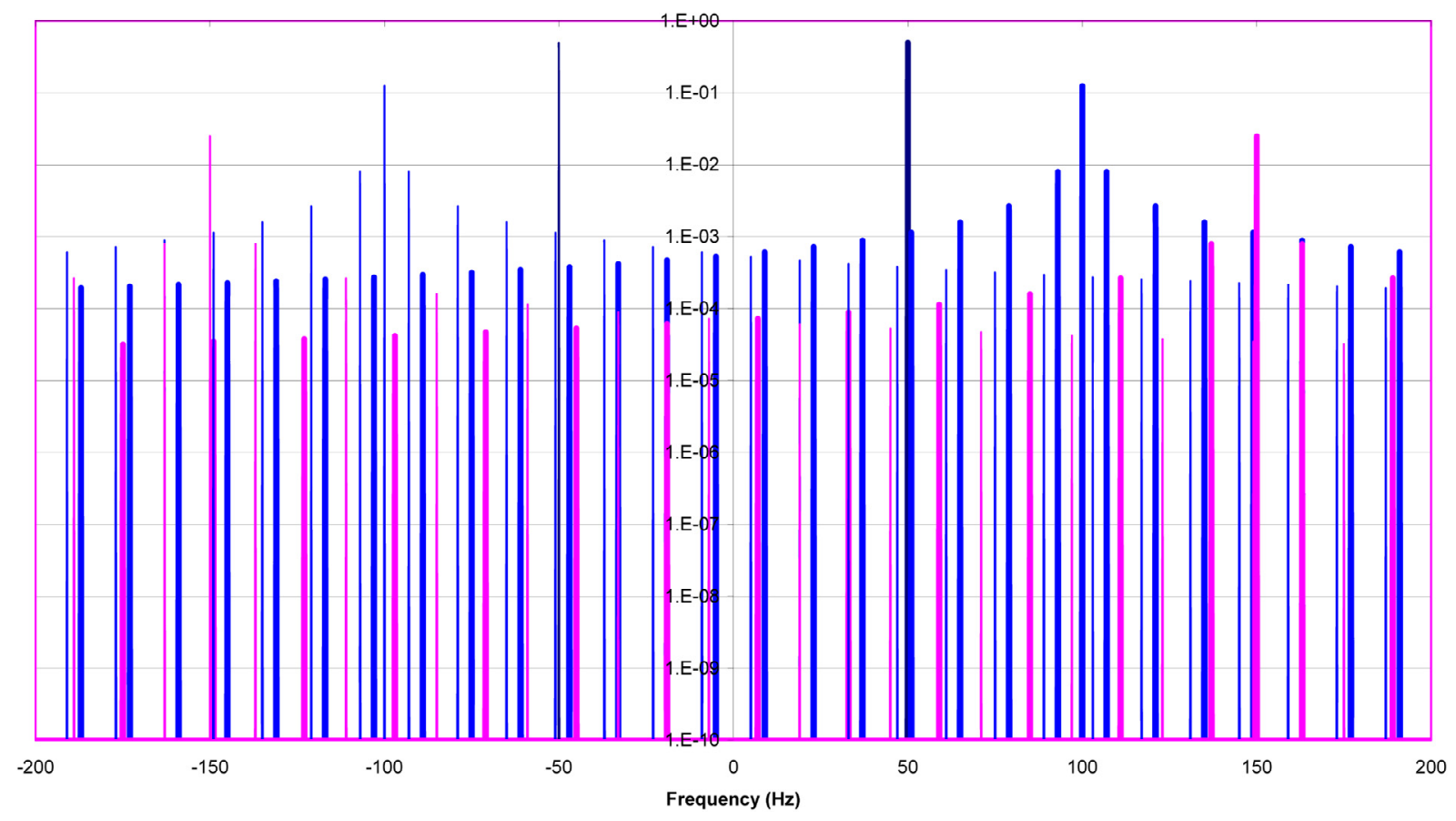

Fig. 1. (Color online) Spectrum of a signal consisting of a fundamental component at $50 \mathrm{~Hz}$ and two fluctuating harmonics of 3rd and 5th orders (observed over an infinite time interval).

\section{Presentation of the method}

\subsection{Condition of application and determined parameters}

Signals that can be analysed by this method consist in a fundamental low frequency (typically $50 \mathrm{~Hz}$ ) component and some fluctuating harmonics. They can be written under the form:

$$
\begin{aligned}
s(t)= & U_{1} \sin \left(2 \pi f_{0} t+\phi_{1}\right)+\sum_{n>1} U_{n}\left[1+k_{n} \operatorname{Mod}_{n}\left(t, \theta_{n}\right)\right] \\
& \times \sin \left(2 \pi n f_{0} t+\phi_{n}\right)
\end{aligned}
$$

where:

$f_{0}$ is the frequency of the fundamental component;

$U_{1}$ its magnitude;

$\phi_{1}$ its phase;

$U_{n}$ the magnitude of $n$th order harmonic;

$\phi_{n}$ its phase;

$k_{n}$ its modulation depth $\left(0 \leqslant k_{n} \leqslant 1\right)$;

$\operatorname{Mod}_{n}\left(t, \theta_{n}\right)$ the modulation waveform of $n$th order harmonic and $\theta_{n}$ the phase of this waveform.

$k_{n}=0$ corresponds to a non modulated signal, $k_{n}=1$, to a "fully" modulated signal. We have defined $\operatorname{Mod}_{n}\left(t, \theta_{n}\right)$ in the most general case as a periodic function that has to fulfill the two following conditions: its mean value should be equal to 0 and its minimal value equal to -1 (see Appendix A).

An example of spectrum of such signals (observed over an infinite time interval) is presented in Figure 1. The component at $50 \mathrm{~Hz}$ is the fundamental one, components at
$100 \mathrm{~Hz}$ and $150 \mathrm{~Hz}$ are the 2nd and the 3th order harmonics and the other components are the modulation components around each harmonic.

Parameters that can be determined by this method are the magnitude and the phase of the fundamental component, magnitudes, phases and modulation depths of all fluctuating harmonics and phases of all modulation waveforms. Parameters that have to be known before applying the method are the frequency of the fundamental component, the orders of all fluctuating harmonics, their modulation frequencies and the shapes of their modulation waveforms (sine, triangle, square, ...). Therefore, this method cannot be used for the analysis of "unknown" signals, measurements on the power grid for example, but can have an application in the calibration of programmable sources that provide reference signals containing fluctuating harmonics. These sources, after calibration, can be considered as reference standards for the traceability of instruments used to check the power grid quality or the immunity of loads that can be connected to the grid.

\subsection{Principle of the method}

The signal to analyse is sampled with a number $N_{s}$ of samples at a sampling frequency $f_{s}$. The parameters to determine are deduced by Fourier analysis.

Indeed equation (1) can also be written as:

$$
\begin{aligned}
s(t)= & U_{1} \sin \left(2 \pi f_{0} t+\phi_{1}\right)+\sum_{n>1} U_{n} \sin \left(2 \pi n f_{0} t+\phi_{n}\right) \\
& +\sum_{n>1} U_{n} k_{n} \operatorname{Mod}_{n}\left(t, \theta_{n}\right) \sin \left(2 \pi n f_{0} t+\phi_{n}\right) .
\end{aligned}
$$


The modulating waveform $\operatorname{Mod}_{n}\left(t, \theta_{n}\right)$ of the $n$th order harmonic can be expressed as Fourier series as follows:

$$
\operatorname{Mod}_{n}\left(t, \theta_{n}\right)=\sum_{m>0} M_{n, m} \sin \left[2 \pi m f_{m o d-n} t+\left(m \theta_{n}+\theta_{n, m}\right)\right]
$$

where $M_{n, m}$ is the $m$ th coefficient of the Fourier series of $\operatorname{Mod}_{n}\left(t, \theta_{n}\right), \theta_{n, m}$ the phase of its $m$ th component (when $\theta_{n}=0$ ) and $f_{m o d-n}$ (assumed to be known) the frequency of $\operatorname{Mod}_{n}\left(t, \theta_{n}\right)$.

Taking into account relation 3 , equation (2) can be written as:

$$
\begin{gathered}
s(t)=U_{1} \sin \left(2 \pi f_{0} t+\phi_{1}\right)+\sum_{n>1} U_{n} \sin \left(2 \pi n f_{0} t+\phi_{n}\right) \\
+\sum_{n>1} \sum_{m>0} U_{n, m}\left\{\sin \left[2 \pi\left(n f_{0}-m f_{\text {mod-n }}\right) t+\phi_{n, m-d i f f}\right]\right. \\
\left.\quad+\sin \left[2 \pi\left(n f_{0}+m f_{\text {mod-n }}\right) t+\phi_{n, m-\text { sum }}\right]\right\}
\end{gathered}
$$

where

$$
U_{n, m}=\frac{U_{n} k_{n} M_{n, m}}{2}
$$

is the magnitude of the $m$ th modulation component of the $n$th fluctuating harmonic and quantities

$$
\begin{aligned}
\phi_{n, m-\text { diff }} & =\phi_{n}-\theta_{n, m}-m \theta_{n}+\frac{\pi}{2} \\
\text { and } \phi_{n, m-\text { sum }} & =\phi_{n}+\theta_{n, m}+m \theta_{n}+\frac{3 \pi}{2}
\end{aligned}
$$

represent phases of modulation components at frequencies $n f_{0}-m f_{\text {mod-n }}$ and $n f_{0}+m f_{\text {mod- } n}$.

All parameters to be determined can be deduced from Fourier analysis. Indeed, for any $n$th order harmonic, quantities $U_{n}, \phi_{n}, U_{n, m}, \phi_{n, m-s u m}$ and $\phi_{n, m \text {-diff }}$ are directly obtained by Fourier Transform of the sampled signal, $M_{m, n}$ and $\theta_{n, m}$ can be theoretically calculated (see relation (3)) as the modulation wave shape is assumed to be known.

Then, the modulation depth $k_{n}$ can be deduced from relation (5) and written as:

$$
k_{n}=2 \frac{U_{m, n}}{U_{n} M_{m, n}}
$$

and phases $\theta_{n}$ of modulation waveforms can be obtained from relations $(6 \mathrm{a})$ or $(6 \mathrm{~b})$ and then given by:

$$
\begin{aligned}
\theta_{n} & =\frac{1}{m}\left[\phi_{n}-\theta_{n, m}-\phi_{n, m-\operatorname{diff}}+\frac{\pi}{2}\right] \\
\text { or } \theta_{n} & =\frac{1}{m}\left[\phi_{n}+\theta_{n, m}-\phi_{n, m-\text { sum }}+\frac{3 \pi}{2}\right] .
\end{aligned}
$$

Calculation can be made for any modulation component (i.e for any value of $m$ ) as well for $k_{n}$ as for $\theta_{n}$. In real measurements application, the first modulation component $(m=1)$ which has the largest magnitude should be chosen for a better signal to noise ratio, using finally:

$$
\begin{aligned}
k_{n} & =2 \frac{U_{1, n}}{U_{n} M_{1, n}} \\
\text { and } \theta_{n} & =\phi_{n}+\theta_{n, 1}-\phi_{n, 1-\text { sum }}+\frac{3 \pi}{2} .
\end{aligned}
$$

Note that in the particular case of square modulating waveforms (taking values +1 for $0 \leqslant t<\frac{1}{2 f_{\text {mod-n }}}$ and -1 for $\left.\frac{1}{2 f_{\text {mod- } n}} \leqslant t<\frac{1}{f_{\text {mod-n }}}\right)$ considered in all numerical simulations presented here (see Chap. III), $M_{1, n}=\frac{4}{\pi}$ and $\theta_{n, 1}=0$, and consequently, $k_{\mathrm{n}}$ and $\theta_{n}$ are calculated from:

$$
\begin{aligned}
k_{n} & =\frac{U_{1, n}}{U_{n}} \frac{\pi}{2} \\
\text { and } \theta_{n} & =\phi_{n}-\phi_{n, 1-\text { sum }}+\frac{3 \pi}{2} .
\end{aligned}
$$

\subsection{Limitation of the Fourier analysis}

The first limitation is that the Shanon condition is not fulfilled for most of the signals analyzed. Indeed, most of modulation waveforms (as square, triangular, saw tooth, ... waveforms) give rise to spectrum component at frequencies far above half the sampling frequency noted $f_{s}$. In the analysis we give in Appendices B and C, an error resulting from a folded component of a spectrum centred around $\pm m f_{s}$ is called $m$ th order aliasing error.

An other limitation comes from that in absence of simple relation between the fundamental component frequency and modulation frequencies, the signal cannot be observed at the same time over an integer number of the fundamental component period and integer numbers of all modulation periods, leading thus to truncature errors.

At last, independently on aliasing or truncature, for certain values of the modulation frequencies, modulation components can overlap some spectral components used in the calculation of the parameters to determine giving also rise to errors. Direct covering occurs when the overlapping modulation component comes directly from the positive part (represented in bold in Fig. 1) of the spectrum. When it comes from the negative part of the spectrum we call it indirect covering.

Applied in such condition, the Fourier analysis can lead to significant errors. An iterative process described below and intended to estimate and correct all these errors has been developed.

\subsection{Iterative process}

The schematic diagram of the iterative process is given in Figure 2. The sampled signal is applied to a Fourier block that

- applies a window to the signal;

- calculates the Fourier transform of the signal;

- determines the magnitudes and phases of the components of the signal and the modulation depth of the fluctuating harmonics.

Let us call $P$ the set of initial values of the parameters to determine and $P_{0}$ the first set of computed values. $P$ and $P_{0}$ are one column matrix which elements are the values 


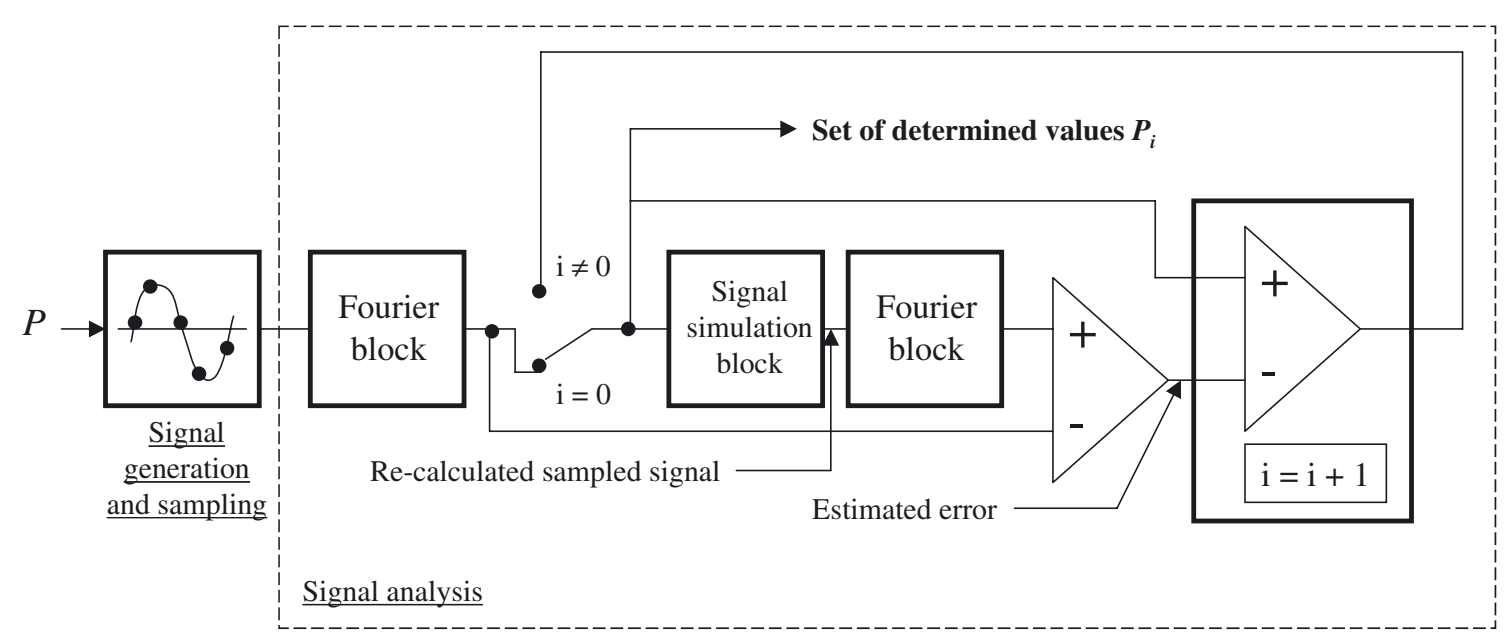

Fig. 2. Schematic diagram of the iterative process.

of these parameters,

$$
P=\left[\begin{array}{c}
U_{1} \\
\phi_{1} \\
\cdot \\
U_{n} \\
\phi_{n} \\
k_{n} \\
\theta_{n} \\
\cdot
\end{array}\right]
$$

A signal simulation block recalculates a new sampled signal from values contained in $P_{0}$ which is applied to the Fourier block that recalculates a new set of values $P_{0}^{\prime}$. As the Fourier analysis can lead to some error (see previous paragraph), values contained in $P_{0}$ can differ from initial values $P$ but the assumption is made that values in $P_{0}$ are relatively close to values in $P$, so that the signal generated from $P_{0}$ can be assumed to be close to the initial signal. In this case, matrix $\varepsilon_{0}=P_{0}^{\prime}-P_{0}$ which represents the exact error made on the determination of values contained in $P_{0}^{\prime}$ that are presumably small, is also an estimator of the error made on the determination of values contained in $P_{0}$. Then, $P-P_{0} \cong P_{0}-P_{0}^{\prime}$, that leads to $P \cong 2 P_{0}-P_{0}^{\prime}=P_{0}-\varepsilon_{0}$.

Let us call $P_{1}=P_{0}-\varepsilon_{0}$ that represents a set of corrected values for the parameters that need to be determined. $P_{1}$ is applied to the signal simulation block and a new set of values $P_{1}^{\prime}$ of the parameters to be determined is delivered at the output of the Fourier block with an estimator $\varepsilon_{1}=P_{1}^{\prime}-P_{0}$ of the error. The process can be repeated and after $p$ iterations we have $P_{p}=P_{(p-1)}-\varepsilon_{(p-1)}$ with an estimator $\varepsilon_{p}$ of the error on $P_{p}$ given by $\varepsilon_{p}=$ $P_{p}^{\prime}-P_{0}$ that should decrease after each iteration.

\section{Results of simulations}

In all simulations, we have considered signals consisting of a fundamental low frequency $(50 \mathrm{~Hz})$ component and only two fluctuating harmonics modulated by square modulating waveforms. Moreover, we have considered only magnitudes and modulation depths. Particularly, in the iterative process we did not take phases into account. Matrix $P$ used in our simulations can then be written

$$
P=\left[\begin{array}{c}
U_{1} \\
\cdot \\
U_{n} \\
k_{n} \\
\cdot
\end{array}\right] .
$$

The signal generation and the sampling systems presented in Figure 2 were replaced by a signal simulation block and furthermore a "differential" block that calculates the difference between the determined values $P_{i}$ and the original values $P$ of the parameters of the analysed signal (effective error) has been added (Fig. 3). Then, the result of each simulation consists in a set of determined values $P_{i}$ with the associated error $\varepsilon_{i}=P_{i}-P$. In the following graphs we have reported only the errors. These errors $\delta_{\text {magn }}$ and $\delta_{\text {mod.depth }}$ have been calculated using $\delta_{\text {magn }}=\frac{U_{n \text {-det }}-U_{n \text {-orig }}}{U_{1 \text {-orig }}}$ for magnitudes and $\delta_{\text {mod.depth }}=k_{n \text {-det }}-k_{n \text {-orig }}$ for modulation depths, where "det" and "orig" in the index refer respectively to determined and original values of the parameter.

\subsection{Analysis of a periodic signal by Fourier transform}

The simulations have been performed for a signal consisting in a fundamental component at $50 \mathrm{~Hz}$ and two fluctuating harmonics of 6 th and 7 th orders which magnitudes are equal to $10 \%$ of the fundamental component and modulation depths equal to 0.1 . The modulation frequency of the 6th order harmonic varies between $1 \mathrm{~Hz}$ and $10 \mathrm{~Hz}$ by steps of $0.1 \mathrm{~Hz}$ and the modulation frequency of the 7 th order harmonic has been set at $1.7 \mathrm{~Hz}$.

This signal is represented by 30000 samples taken at a sampling frequency of $3 \mathrm{kHz}$. These values are compatible with precision measurement instruments available on the 


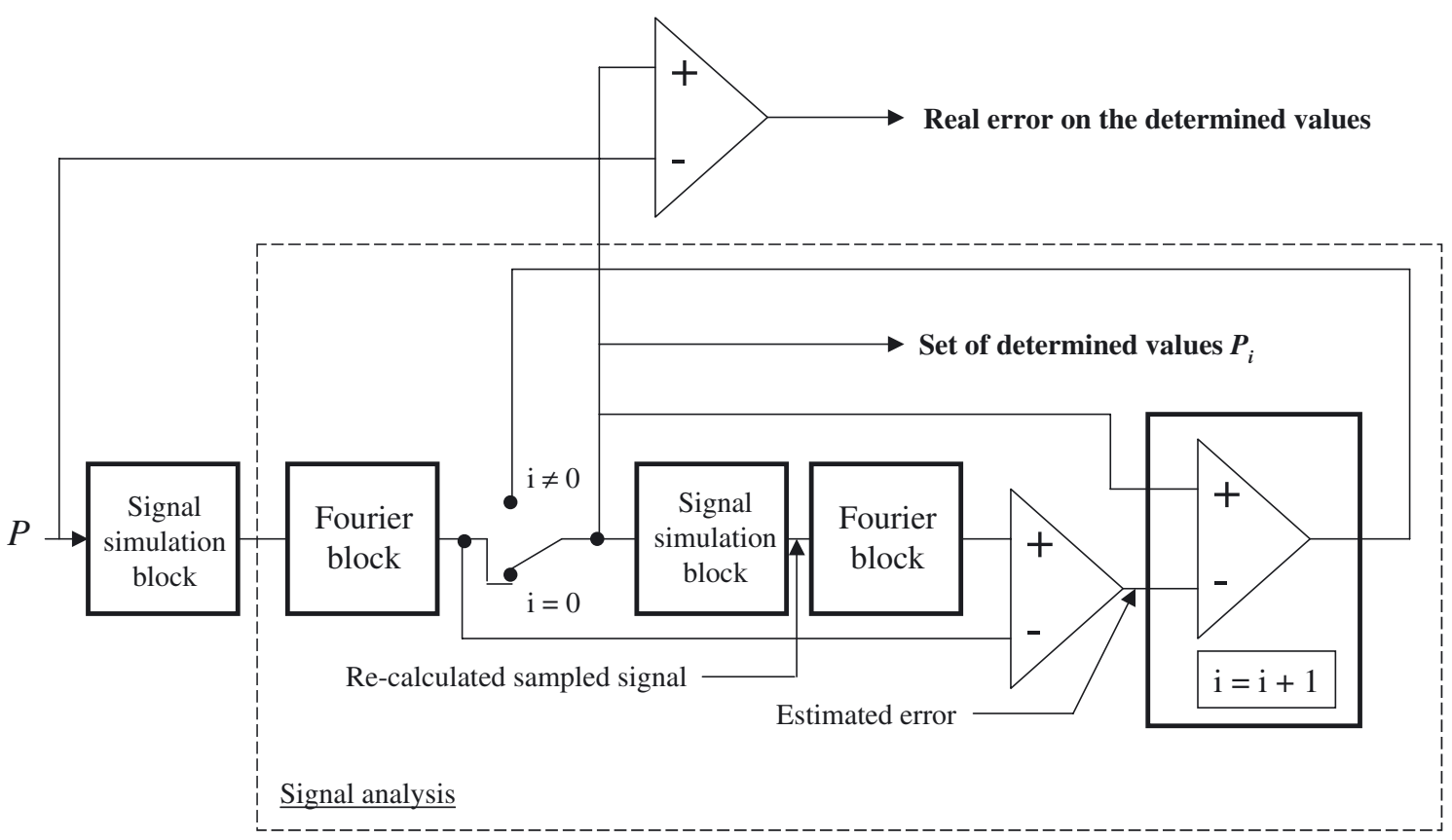

Fig. 3. Schematic diagram of the performed simulations.

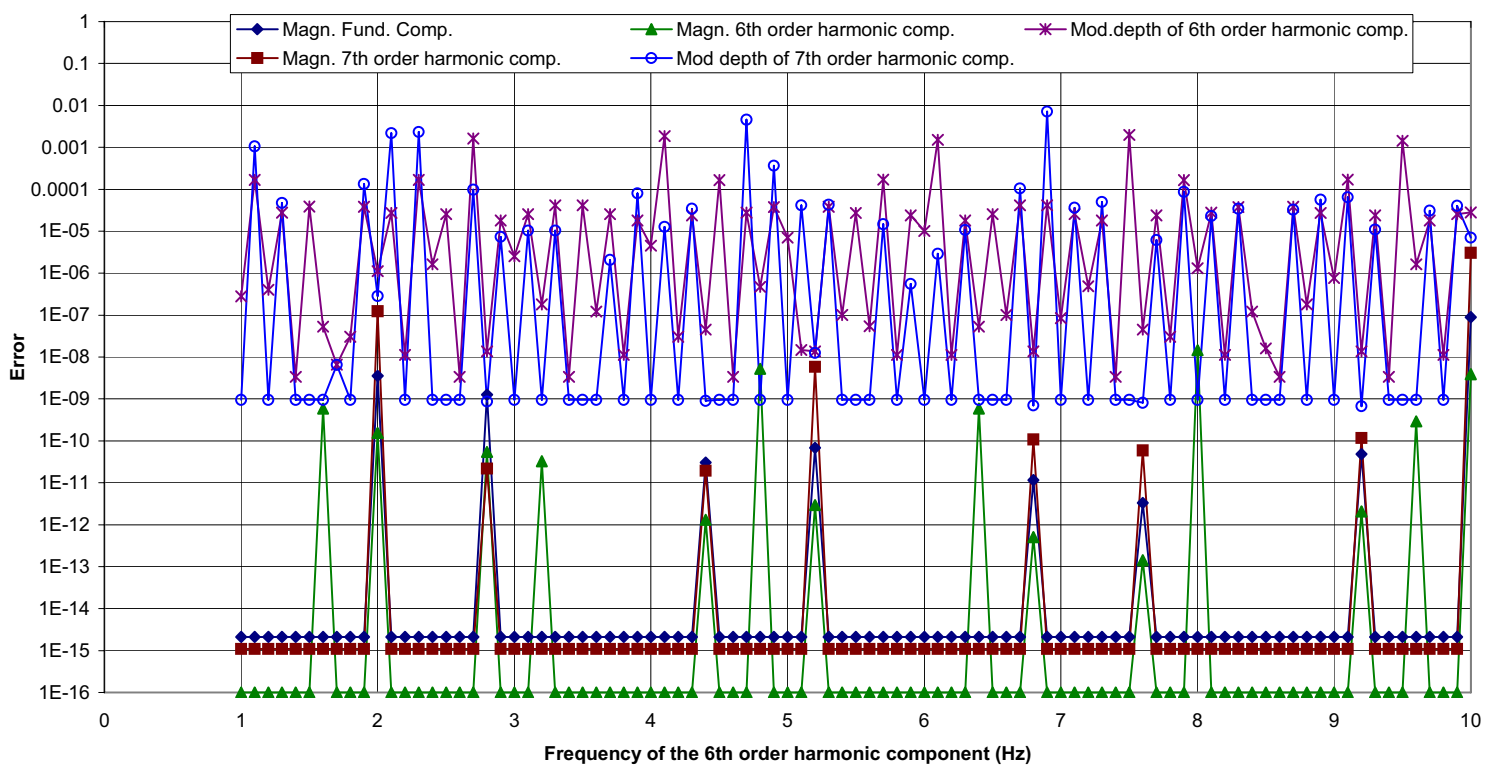

Fig. 4. (Color online) Error on the determined values of the parameters of the analysed signal as functions of the modulation frequency $f_{m 6}$ of the 6 th order harmonic; $f_{0}=50 \mathrm{~Hz}, f_{6}=300 \mathrm{~Hz}, f_{7}=350 \mathrm{~Hz}, U_{6}=U_{7}=10 \%$ of fund., $k_{6}=k_{7}=0.1$, $f_{m 7}=1.7 \mathrm{~Hz}, f_{s}=3 \mathrm{kHz}, 30000$ samples, 0 iteration.

market. The observation time interval is of $10 \mathrm{~s}$, it leads to a frequency resolution of the Fourier transform of $0.1 \mathrm{~Hz}$, and corresponds to 500 periods of the fundamental component, 17 modulation periods of the 7 th harmonic and integer numbers of the modulation period of the 6 th order harmonic. Then, in all considered cases, the analysed signal, which is perfectly periodic, is observed over an integer number of periods.

Errors on the determined parameters (magnitude of the fundamental component, magnitudes of the 6th and 7th order harmonics and their modulation depths), ob- tained without any iteration, are given as functions of the modulation frequency of the 6th order harmonics in Figure 4 . Curves relative to magnitudes show a very low error level (no more than a few parts in $10^{15}$ ) with a few spikes corresponding to large errors (up to some parts in $10^{6}$ ). Modulation depth curves are much less regular and never take values lower than a few parts in $10^{9}$.

All major errors come from the overlapping of components to be determined or used in their calculation by modulation components due to direct or indirect covering or to aliasing. A detailed analysis of errors observed on the 


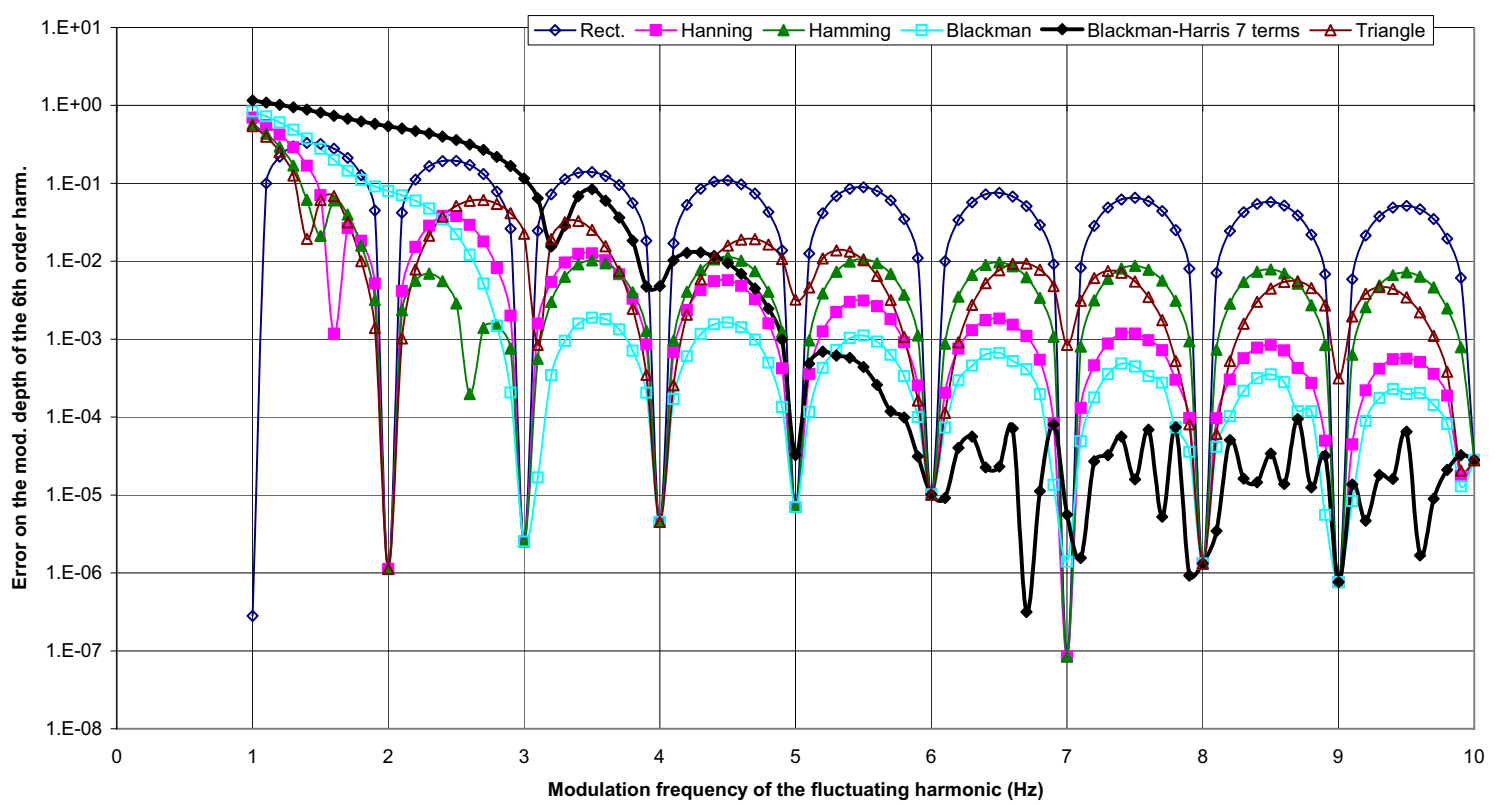

Fig. 5. (Color online) Error on the modulation depth for various windows as a function of the modulation frequency; $f_{0}=50 \mathrm{~Hz}$, $f_{6}=300 \mathrm{~Hz}, U_{6}=10 \%$ of fund., $k_{6}=0.1, f_{s}=3 \mathrm{kHz}, 3000$ samples, 0 iteration, zero padding $=\times 10$.

magnitude of the 6th order harmonic is presented in Appendix B. A similar analysis has been made for the other parameters and all errors could be explained in the same way. It can be shown (see Appendix C) for example, that for simulation conditions given above, there is always an overlapping of the first modulation component (the first modulation component is taken into account in the calculation of the modulation depth) by some other component of the spectrum, whatever the order of the fluctuating harmonic and the modulation frequency. This overlapping leads to an error on modulation depth of about 1 part in $10^{9}$ in the best cases which is well illustrated in Figure 4. Nevertheless, other simulation conditions can lead to errors on modulation depths as low as a few parts in $10^{15}$.

Simulations presented above have shown that for periodic signals observed over an integer number of periods, Fourier transform allows accurate characterisation of signals containing fluctuating harmonics as long as no overlapping of useful components for calculation occurs.

\subsection{Analysis of a signal over a non integer number of periods}

Errors occur when a signal is observed over a time interval that does not correspond to an integer number of periods. They can be reduced by applying appropriate windows. Some windows among the more currently used have been tested to determine the most convenient one for our application.

\subsubsection{Simulation conditions}

In order to make observations over a non integer number of modulation periods when the modulation frequency varies between $1 \mathrm{~Hz}$ and $10 \mathrm{~Hz}$, the number of samples has been reduced to 3000 , which led to an observation time interval of $1 \mathrm{~s}$ for a sampling frequency of $3 \mathrm{kHz}$. Then all modulating frequencies which values cannot be expressed as integer numbers of $\mathrm{Hz}$ correspond to signals that are not observed over an integer number of periods. As the $1 \mathrm{~s}$ observation time interval leads to a frequency resolution of $1 \mathrm{~Hz}$, the required $0.1 \mathrm{~Hz}$ resolution has been reached by using zero padding technique consisting in adding $9 \times 3000$ "zeros" after the 3000 samples before calculating the Fourier transform (effective number of samples used in the calculation increased by a factor 10 leading to a frequency resolution 10 times better).

\subsubsection{Improvement due to windowing}

The analysed signal consisted in a fundamental component at $50 \mathrm{~Hz}$ and only one 6th order harmonic with a magnitude equal to $10 \%$ of the fundamental component and a modulation depth equal to 0.1 .

Several windows have been applied to the signal and their effects on the determination of the magnitudes and modulation depths have been studied. Figure 5 presents this effect on the modulation depth of the fluctuating harmonic. The rectangular window gives good results only when the signal is observed over an integer number of periods. Otherwise it leads to large errors that nevertheless decrease slowly when the number of observed periods of the signal increases. The other windows lead in most cases to much smaller errors. The best results are generally obtained with the Blackman-Harris 7 terms (BH7) window provided the number of periods of the signal that are observed is larger than 6 . Compared to the rectangular window, the error is in most cases 100 to 1000 times smaller. 


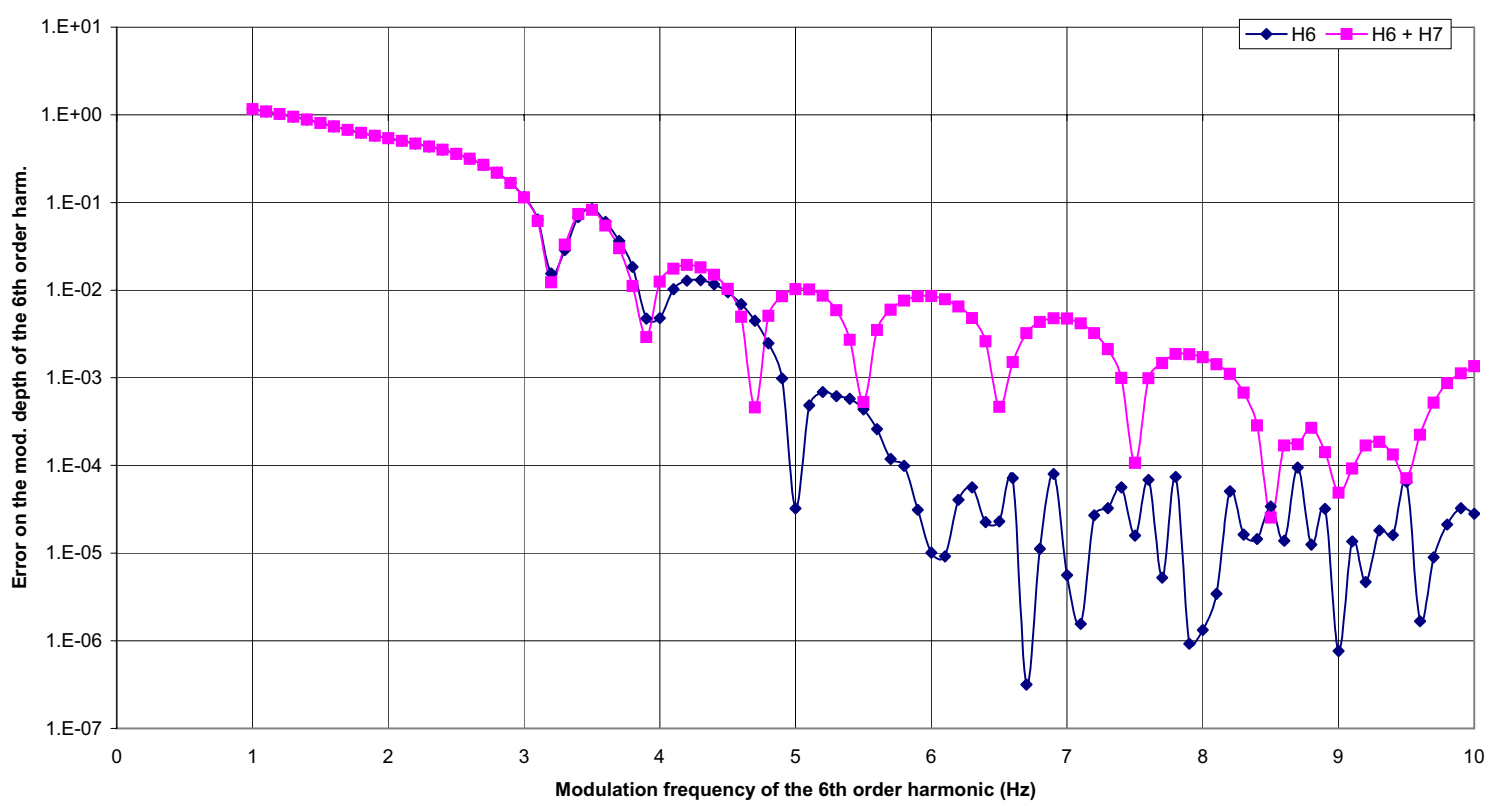

Fig. 6. (Color online) Error on the modulation depth of the 6th order harmonic as a function of its modulation frequency with $f_{s}=3 \mathrm{kHz}, 3000$ samples, 0 iteration, zero padding $=\times 10$. Signal $1(\mathrm{H} 6): f_{0}=50 \mathrm{~Hz}, f_{6}=300 \mathrm{~Hz}, U_{6}=10 \%$ of fund., $k_{6}=0.1 ;$ Signal $2(\mathrm{H} 6+\mathrm{H} 7): f_{0}=50 \mathrm{~Hz}, f_{6}=300 \mathrm{~Hz}, f_{7}=350 \mathrm{~Hz}, U_{6}=U_{7}=10 \%$ of fund., $f_{m 7}=9 \mathrm{~Hz}, k_{6}=k_{7}=0.1$.

The efficiency of the window nevertheless depends on the characteristics of the signal. We have compared errors observed with the previous signal and another signal containing two fluctuating harmonics using in both cases the BH7 window. Results are given in Figure 6. It clearly appears that when the number of periods of the signal observed is larger than 6 , the error for the signal with only one harmonic is generally 10 to 100 times smaller than the error for the signal with two harmonics.

\subsubsection{Conclusion}

Then, in all the following simulations, the $\mathrm{BH} 7$ window has been used and the observation time interval set at $5 \mathrm{~s}$ (15000 samples at a sampling frequency of $3 \mathrm{kHz}$ ) therefore the BH7 window is efficient for all modulation frequencies higher than $1.2 \mathrm{~Hz}$. In these conditions, signals containing modulation frequencies that are not multiples of $0.2 \mathrm{~Hz}$ (1/observation time interval) are not observed over an integer number of periods. The $0.1 \mathrm{~Hz}$ resolution has been obtained by adding 15000 "zeros" after the 15000 samples.

Nevertheless, the error remains generally significant despite the improvement due to the use of a window. In order to reduce this error, the iterative process described above has been applied.

\subsection{Effect of the iterative process}

Figure 7 shows the error on the modulation depth of the 7 th order harmonic remaining after $0,1,2,3,5$ and 10 iterations for a signal consisting of a fundamental component at $50 \mathrm{~Hz}$, a 6th order harmonic fluctuating at frequencies between $1 \mathrm{~Hz}$ and $10 \mathrm{~Hz}$ by steps of $0.1 \mathrm{~Hz}$ and a 7 th order harmonic fluctuating at $1.7 \mathrm{~Hz}$. The magnitudes of both harmonics are equal to $10 \%$ of the fundamental component and the modulation depths are equal to 0.1. It clearly appears that the error is reduced after each iteration, and after 10 iterations it is never larger than a few parts in $10^{16}$. Remaining errors on all determined parameters after 10 iterations are plotted in Figure 8. It appears that they are all of the same order.

As a conclusion, the iterative process reduces the calculation error to an insignificant level compared to uncertainties related to $\mathrm{AC}$ voltage and current measurement instruments (a few parts in $10^{6}$ in the best cases).

\subsection{Influence of the characteristics of the signal (magnitude, modulation depth and orders of harmonics)}

Results presented in previous paragraphs have been obtained for signals containing harmonics with a magnitude equal to $10 \%$ of the fundamental component and a modulation depth equal to 0.1 . We are now focussing on the influence of the value of these parameters on the efficiency of the method. All results presented in this paragraph have been obtained after 10 iterations.

Influence of magnitude of harmonics on the remaining error is presented in Figure 9. Tested signals were similar to signals previously analysed, with only some changes in the magnitudes of harmonics. The modulation depth was kept equal to 0.1 for both harmonics. It appears that the error on the modulation depth of the 7 th order harmonic is insignificant in most cases, and almost always smaller 


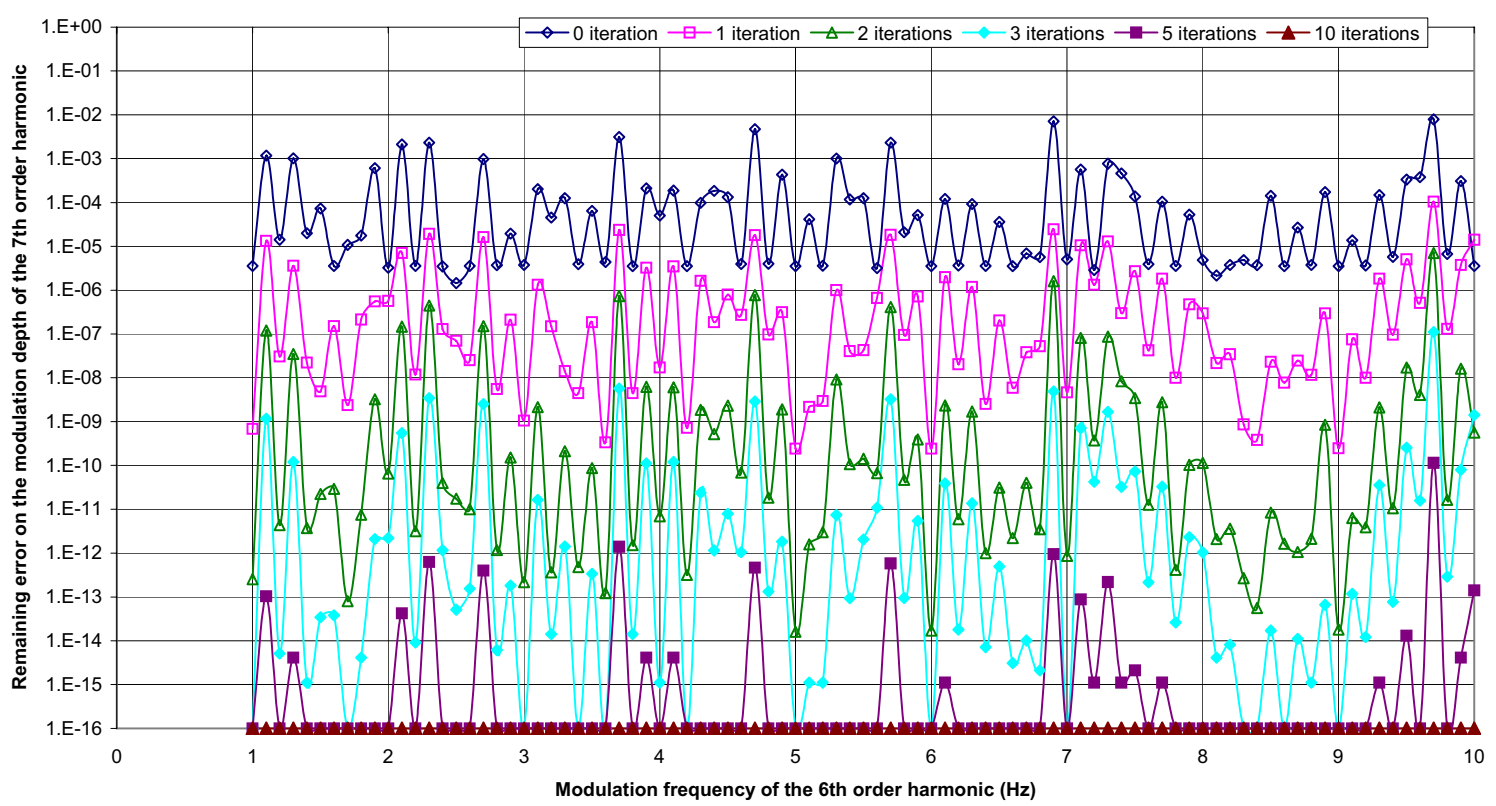

Fig. 7. (Color online) Remaining relative error on the modulation depth of the 7 th order harmonic after $0,1,2,3,5$ and 10 iterations for a signal analysed with a Blackman-Harris 7 terms window. $f_{0}=50 \mathrm{~Hz}, f_{6}=300 \mathrm{~Hz}, f_{7}=350 \mathrm{~Hz}, U_{6}=U_{7}=$ $10 \%$ of fund., $f_{m 7}=1.7 \mathrm{~Hz}, k_{6}=k_{7}=0.1, f_{s}=3 \mathrm{kHz}, 15000$ samples, zero padding $=\times 2$.

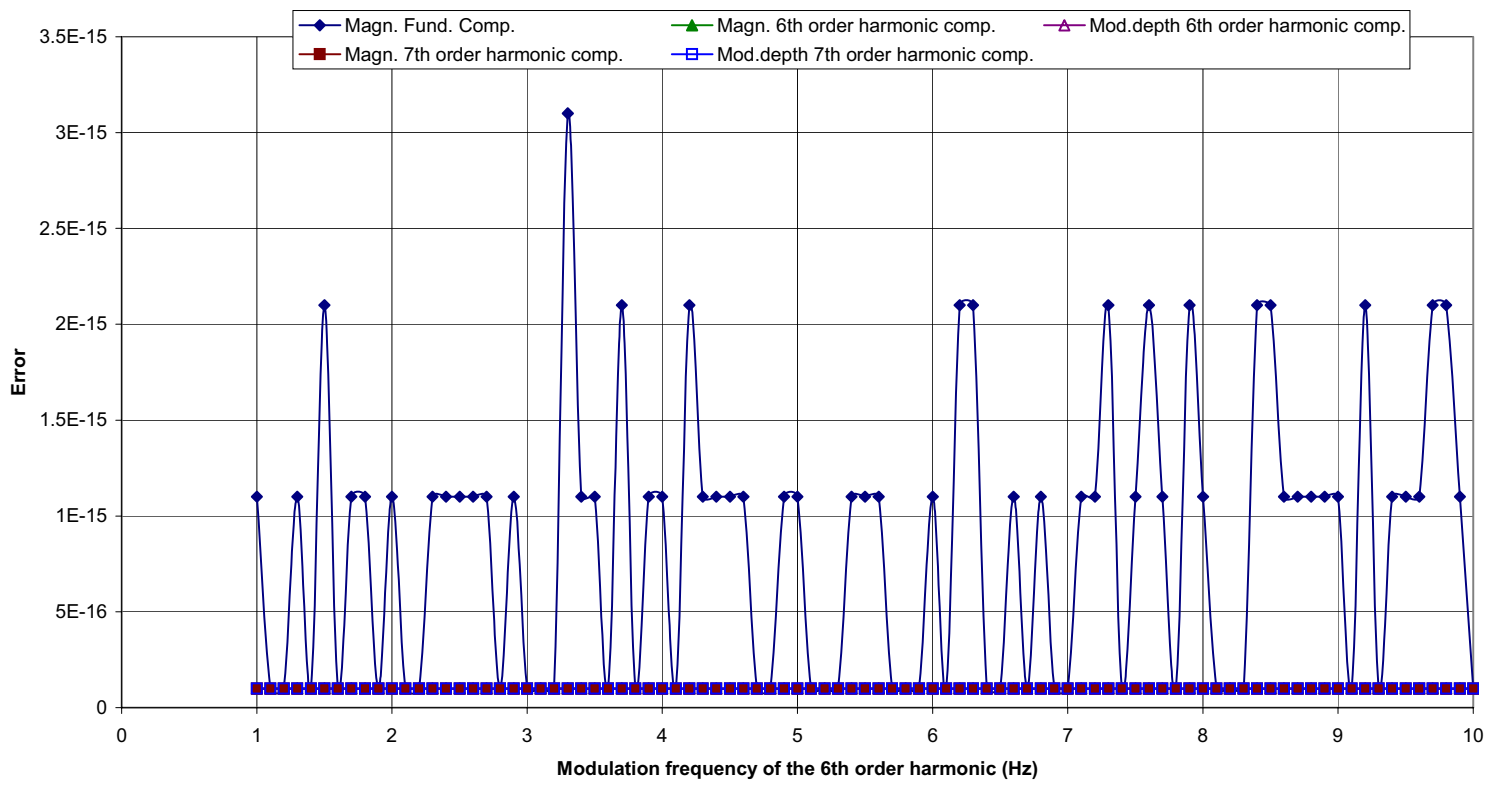

Fig. 8. (Color online) Remaining relative error on all determined parameters after 10 iterations for a signal analysed with a Blackman-Harris 7 terms window. $f_{0}=50 \mathrm{~Hz}, f_{6}=300 \mathrm{~Hz}, f_{7}=350 \mathrm{~Hz}, U_{6}=U_{7}=10 \%$ of fund., $f_{m 7}=1.7 \mathrm{~Hz}, k_{6}=k_{7}=0.1$, $f_{s}=3 \mathrm{kHz}, 15000$ samples, zero padding $=\times 2$.

than 1 part in $10^{10}$. The histogram (Fig. 10), showing the percentage of simulations leading to a given error interval, shows that errors greater than 1 part in $10^{10}$ start to appear only when the ratio between the magnitudes of the harmonics is approximately 10 or larger. When this ratio increases, the number of simulations leading to errors greater than a few parts in $10^{10}$ increases and reaches more than $25 \%$ for a value of 100 (U6-10\%_U7-0.1\%). The number of simulations leading to errors greater than 0.01 ( $10 \%$ of the modulation depth of the analysed signals) is relatively low (about 1\% for a ratio of 10 and $4 \%$ for a ratio of 100). Similar observations have been made for all other parameters. It is interesting to note that very low errors have been observed as well when the magnitude of both components was equal to $10 \%$ of the fundamental as when it was equal to $0.1 \%$. The ratio between the magnitudes is then much more influent than their absolute values.

Similar signals have been used again to test the influence of the modulation depth. Magnitude of harmonics 


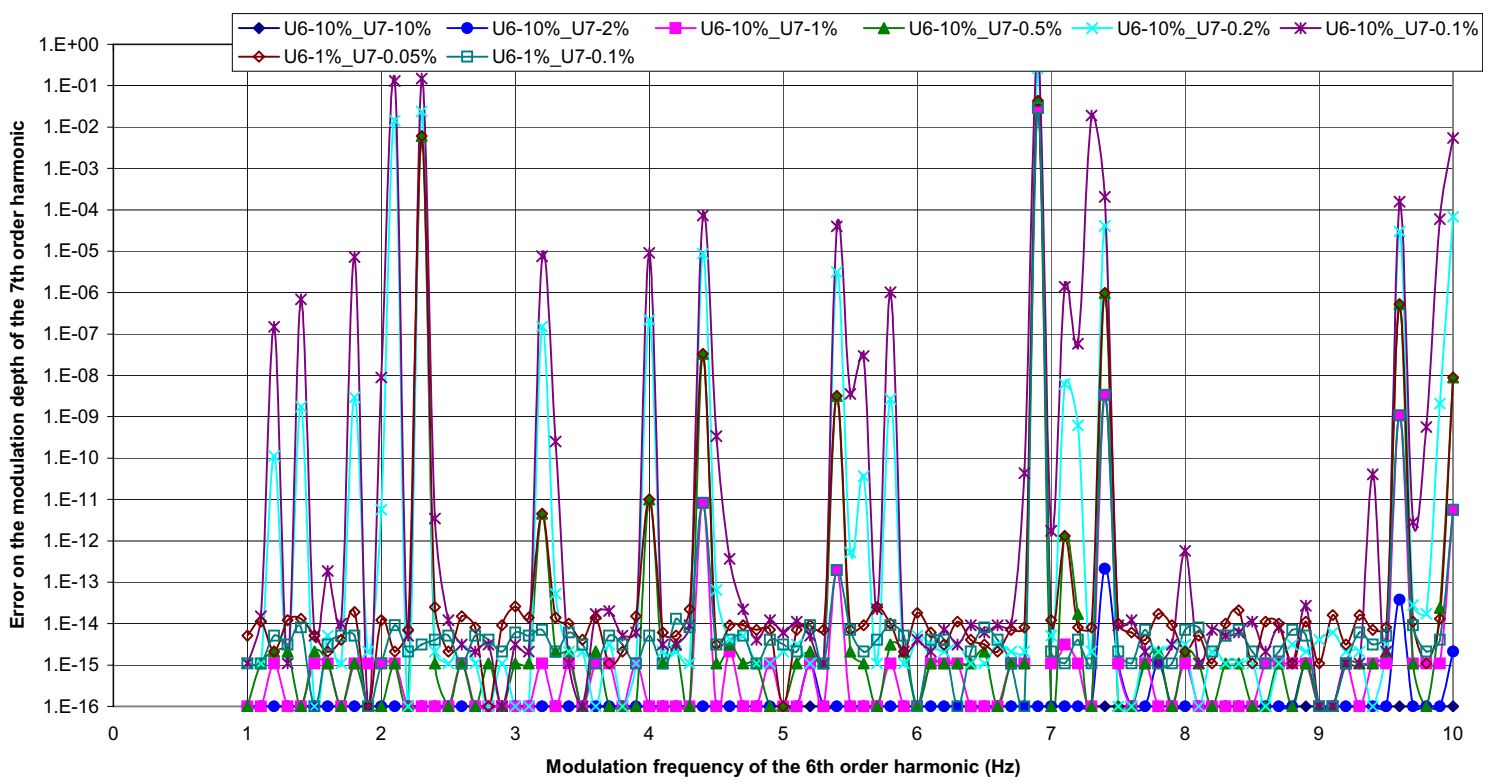

Fig. 9. (Color online) Influence of magnitudes of the harmonic components on the error remaining after 10 iterations for a signal analyzed with the Blackman-Harris 7 terms window. $f_{0}=50 \mathrm{~Hz}, f_{6}=300 \mathrm{~Hz}, f_{7}=350 \mathrm{~Hz}, f_{m 7}=1.7 \mathrm{~Hz}, k_{6}=k_{7}=0.1$. $f_{s}=3 \mathrm{kHz}, 15000$ samples, zero padding $=\times 2$.

Error on the modulation depth of the 7th order harmonic

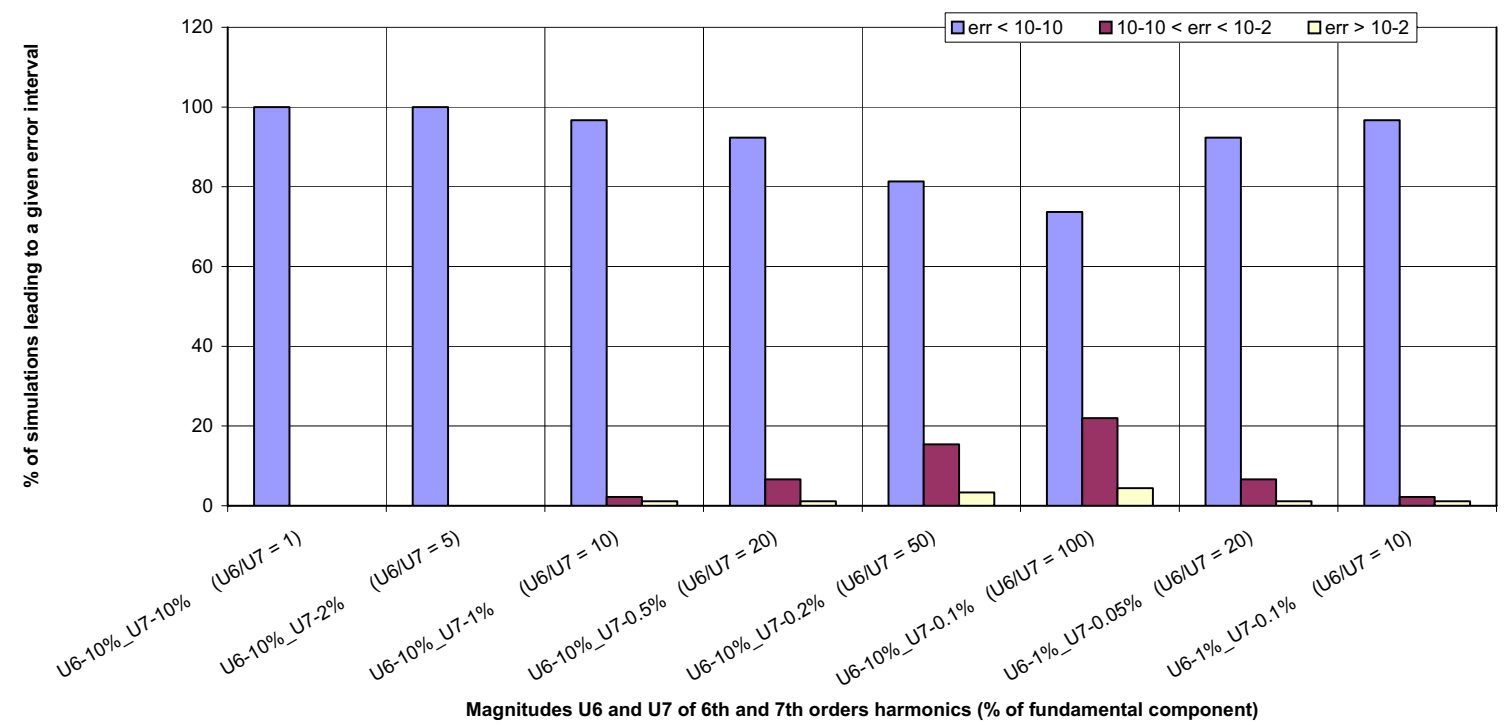

Fig. 10. (Color online) Influence of magnitudes of the harmonic components on the error remaining after 10 iterations (histogram). $f_{0}=50 \mathrm{~Hz}, f_{6}=300 \mathrm{~Hz}, f_{7}=350 \mathrm{~Hz}, f_{m 7}=1.7 \mathrm{~Hz}, k_{6}=k_{7}=0.1 . f_{s}=3 \mathrm{kHz}, 15000$ samples, zero padding $=\times 2$.

has been set to $10 \%$ of the fundamental component. Results of simulations are plotted in Figure 11. The only cases where errors greater than $10^{-10}$ have been observed correspond to large ratios between modulation depths (10 and more). At the same time, small errors have been obtained with modulation depth as low as 0.0001 for both harmonics (not shown in the graphs).

The same results are also presented under the form of histogram in Figure 12. We have defined 4 error intervals: fewer than 1 part in $10^{10}$ errors have been considered irrelevant; errors ranging from 1 part in $10^{10}$ to 1 part in $10^{4}$ (error smaller than 10\% of the measured value in the worst case) can be considered acceptable for application in the frame of real measurements; errors ranging from 1 part in $10^{4}$ to 0.1 that are of the same order as measured values and at last errors larger than 0.1 are totally unacceptable. It appears that for ratio between modulation depths up to 5, all our simulations led to insignificant errors. When these ratios reach 10 , only very small numbers of simulations (about $3 \%$ ) led to errors greater than 1 part in $10^{10}$. A non negligible number (4\% of simulations) of errors of the same order as the measured quantity have been observed for a ratio equal to 100. Errors larger than 0.1 have been found only for signals with high ratios (100) between 


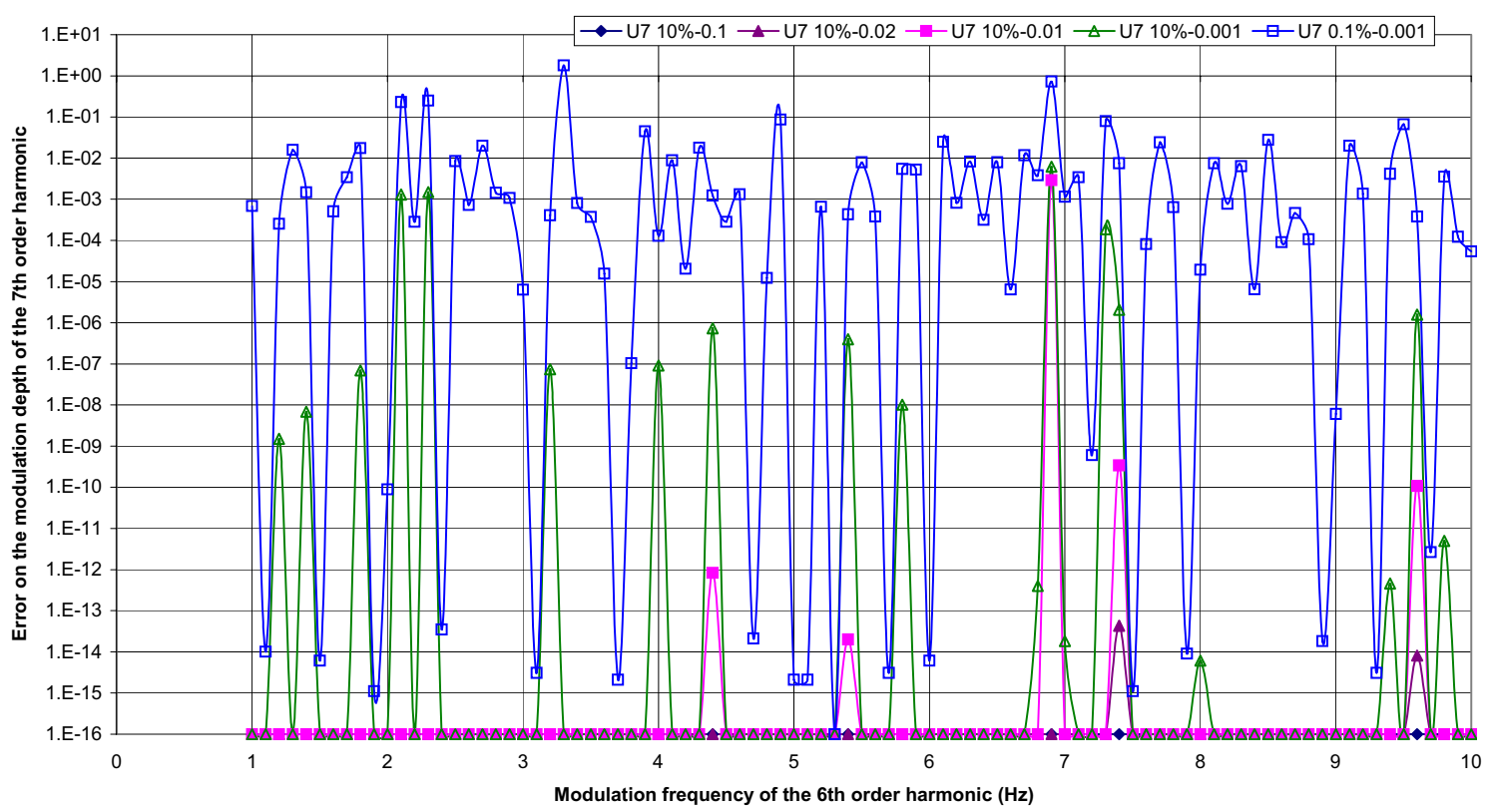

Fig. 11. (Color online) Influence of modulation depths of the harmonic components on the error remaining after 10 iterations for a signal analyzed with the Blackman-Harris 7 terms window. $f_{0}=50 \mathrm{~Hz}, f_{6}=300 \mathrm{~Hz}, f_{7}=350 \mathrm{~Hz}, U_{6}=10 \%$ of fund., $k_{6}=0.1, f_{m 7}=1.7 \mathrm{~Hz} . f_{s}=3 \mathrm{kHz}, 15000$ samples, zero padding $=\times 2$.

Error on the modulation depth of the 7 th order harmonic

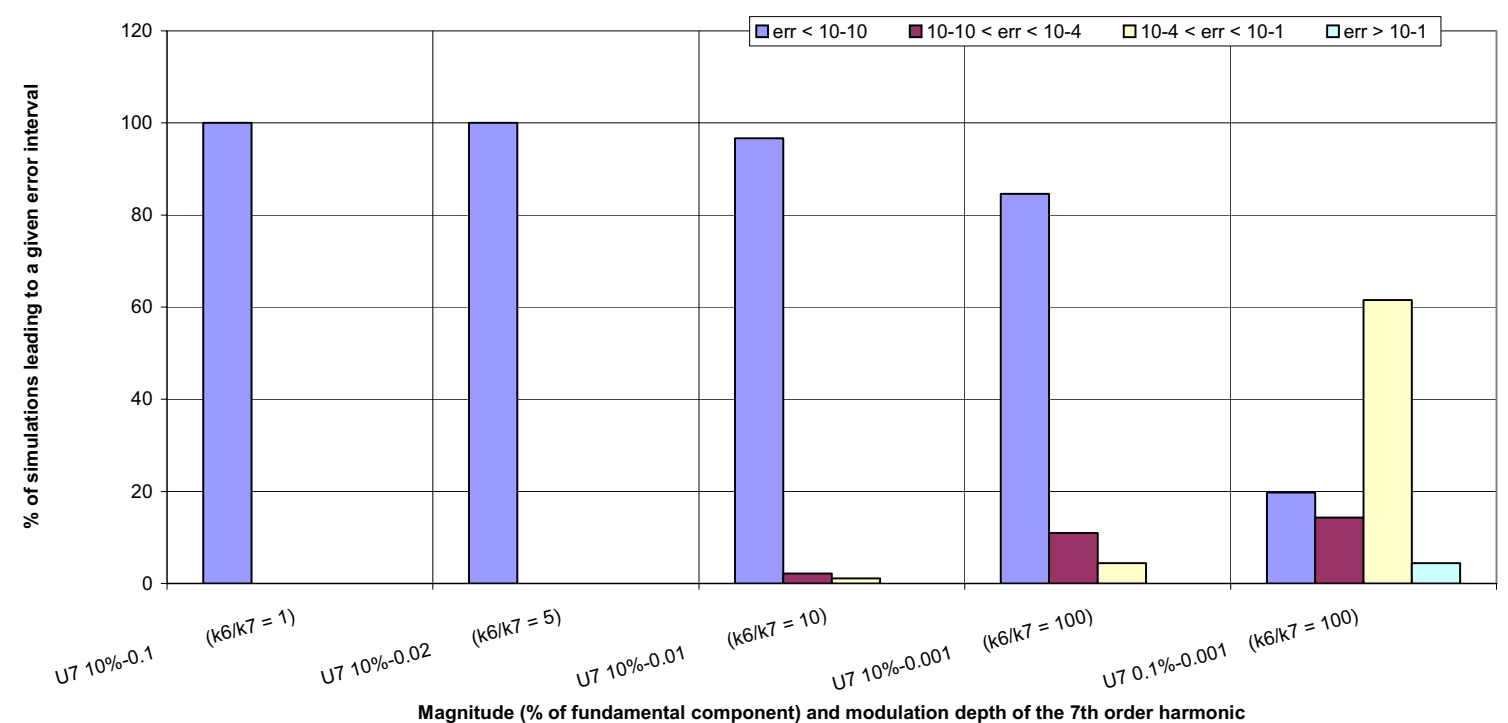

Fig. 12. (Color online) Influence of modulation depths of the harmonic components on the error remaining after 10 iterations (histogram). $f_{0}=50 \mathrm{~Hz}, f_{6}=300 \mathrm{~Hz}, f_{7}=350 \mathrm{~Hz}, U_{6}=10 \%$ of fund., $k_{6}=0.1, f_{m 7}=1.7 \mathrm{~Hz} . f_{s}=3 \mathrm{kHz}, 15000 \mathrm{samples}$, zero padding $=\times 2$.

magnitudes and between modulation depths at the same time. Therefore, a similar conclusion can be drawn for the influence of modulation depth as for the influence of magnitude.

In order to evaluate the performance of the method in unfavourable conditions, we have tested signals such as the product of the ratio between magnitudes multiplied by the ratio between modulation depths is equal to 100 (see Tab. 1). Results are presented under the form of an histogram in Figure 13. The vast majority of simulations led to errors smaller than 1 part in $10^{10}$. The number of simulations leading to errors of the same magnitude as the measured quantity $\left(10^{-4}<e<0.1\right)$ is relatively small (4\% or less depending on the cases) and larger errors appear only for ratio between magnitudes of 20 and above. Then, ratio between magnitudes turns out to be more influent than ratio between modulation depths.

Our simulations showed also that when the orders of the harmonics are very different from each other, the resulting error for signals having large ratios between 
Table 1. Magnitudes and modulation depths of signals used to test the method in unfavorable cases.

\begin{tabular}{cc|cc|ccc}
\hline \multicolumn{2}{c|}{ 6th order harmonic } & \multicolumn{2}{|c|}{ 7th order harmonic } & \multicolumn{3}{c}{ Ratio } \\
\hline Magnitude & Mod. depth & Magnitude & Mod. depth & Magnitude & Mod. depth & Prod. \\
\hline 10 & 0.1 & 0.1 & 0.1 & 100 & 1 & 100 \\
10 & 0.1 & 0.5 & 0.02 & 20 & 5 & 100 \\
10 & 0.1 & 1 & 0.01 & 10 & 10 & 100 \\
10 & 0.1 & 5 & 0.002 & 2 & 50 & 100 \\
10 & 0.1 & 10 & 0.001 & 1 & 100 & 100 \\
\hline
\end{tabular}

Error on the modulation depth of the 7th order harmonic

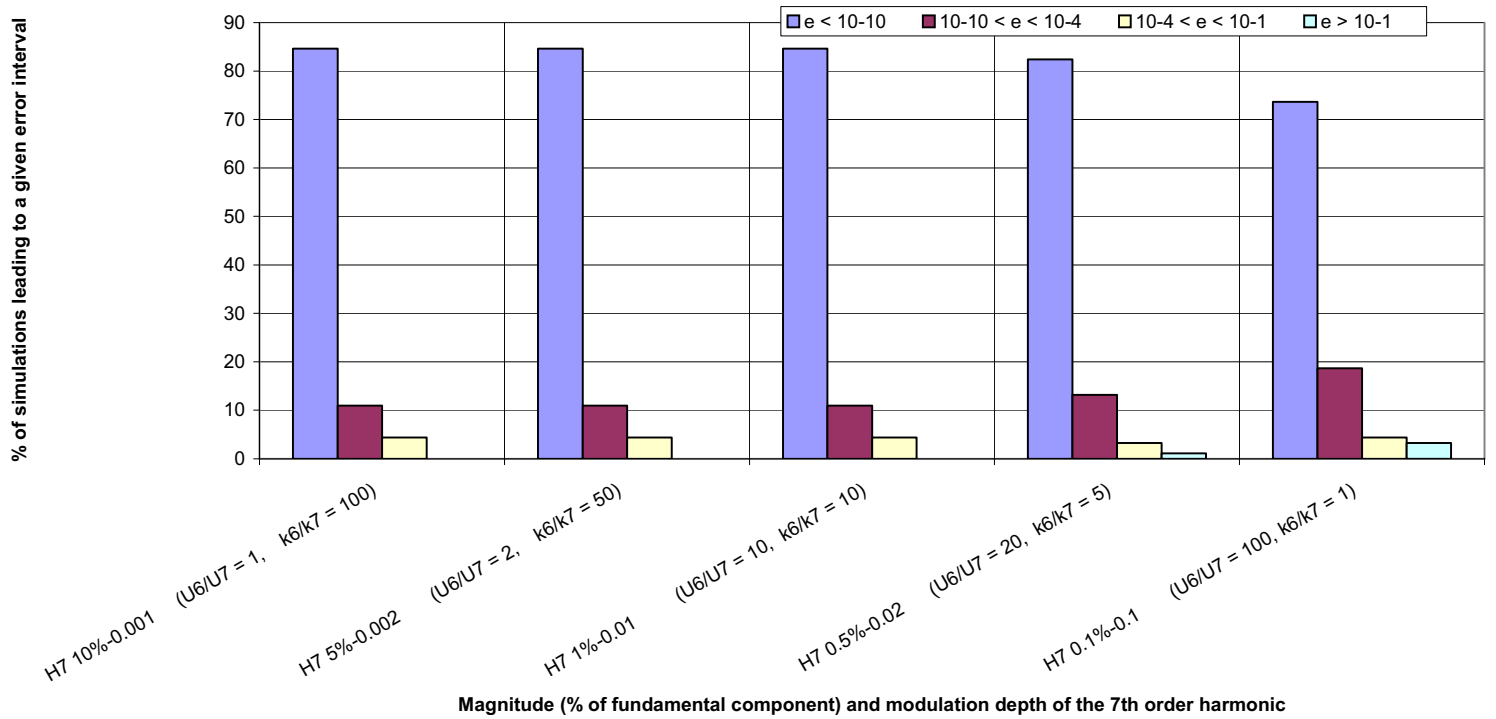

Fig. 13. (Color online) Performance of the method in unfavorable cases. $f_{0}=50 \mathrm{~Hz}, f_{6}=300 \mathrm{~Hz}, f_{7}=350 \mathrm{~Hz}, U_{6}=10 \%$ of fund., $k_{6}=0.1, f_{m 7}=1.7 \mathrm{~Hz} . f_{s}=3 \mathrm{kHz}, 15000$ samples, zero padding $=\times 2$.

harmonic amplitudes and/or modulation depths is generally smaller. The reason is that the magnitude of modulation components relative to one harmonic that can overlap the other harmonic or the component used in the calculation of its modulation depth decreases when the distance between both harmonics increases leading finally to a smaller error. Results are presented in Figure 14. Magnitudes of harmonics are $10 \%$ and $1 \%$ of the fundamental component and modulation depths equal to 0.1 and 0.01. The order of the first harmonic is 5 for all signals and the order of the second harmonic is $6,10,15,20$ and 25 depending on the simulation. Growth of the number of simulations leading to negligible errors when the distance between harmonics increases is well illustrated in Figure 15. On the other hand the number of simulations leading to an error greater than 0.001 (10\% of the measured quantity) becomes insignificant (no more than 1\%) when the orders of the considered harmonics differ by 5 or more.

As a conclusion, this method leads to small errors, generally of the order of a few parts in $10^{15}$ and rarely greater than 1 part in $10^{10}$, after 10 iterations when the BlackmanHarris 7 terms window is used provided the analysed signal is observed over a time interval greater than 6 periods of the modulating waveforms. The best efficiency is obtained when present harmonic components are far from each other in the spectrum of the signal and their magnitudes and modulation depths are of the same orders. When ratios between magnitudes or modulation depths become too large, our simulations led to resulting errors that can sometimes remain at a non acceptable level even after 10 iterations. We believe that taking phases into account in the estimation of the error in the iterative process should allow a finer approximation of the analyzed signal and, as a consequence, could still improve the performance of the method in such conditions.

\section{Conclusion}

A new approach for the analysis of signals containing fluctuating harmonics has been developed. It is based on the Fourier transform, uses observation windows and an original iterative process. It can be applied to the calibration of certain reference standards which can then be used to ensure the traceability of instruments used for power quality measurements.

The method has been evaluated by means of simulations in the particular case of signals containing two harmonic components modulated by square waveforms. In our simulations, we considered only the magnitude of the 


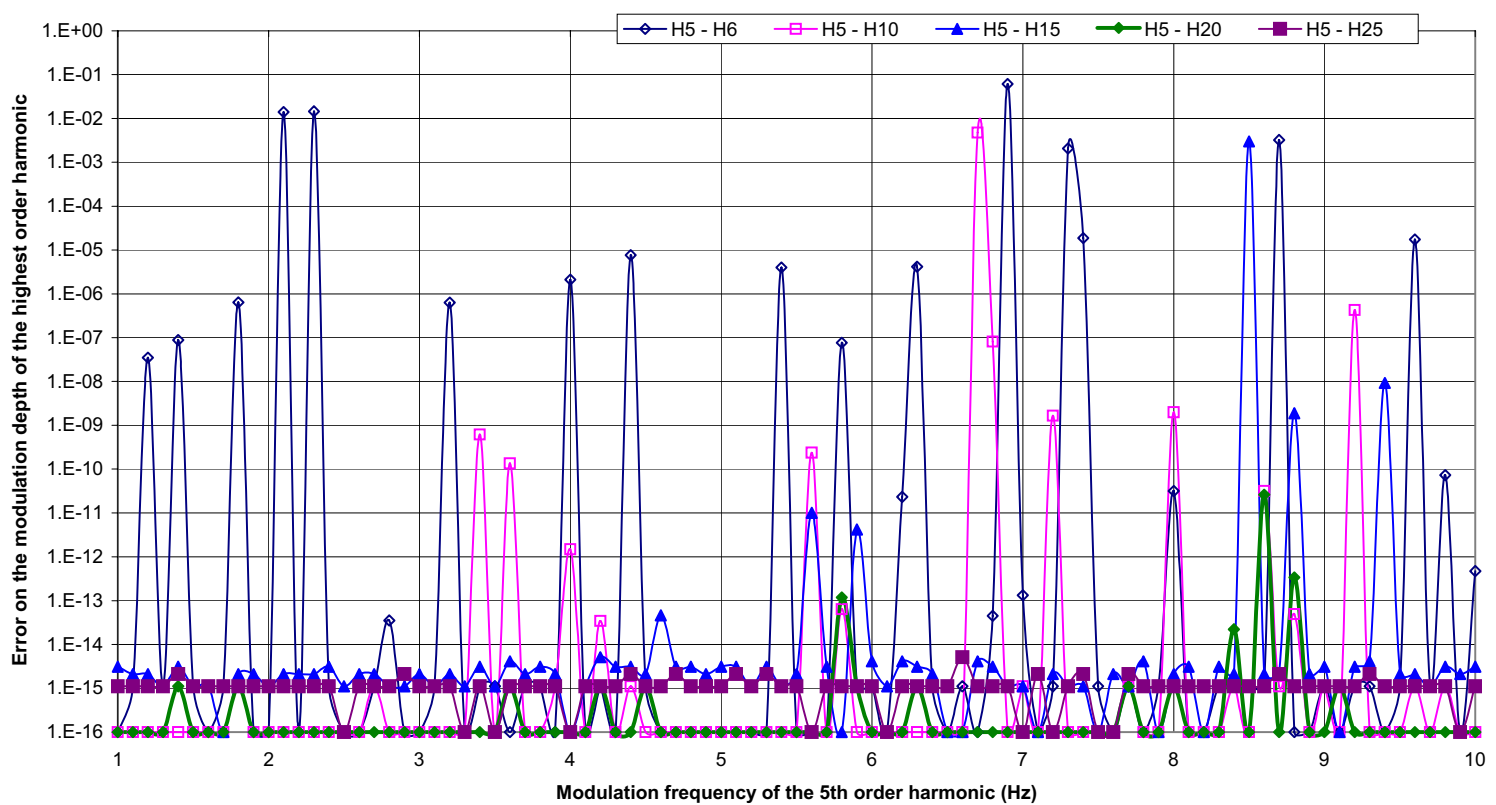

Fig. 14. (Color online) Influence of orders of the harmonic components on the error remaining after 10 iterations for a signal analyzed with the Blackman-Harris 7 terms window. $f_{0}=50 \mathrm{~Hz}$, magnitudes of harmonics: $U_{5}=10 \%$ and $U_{6,10,15,20,25}=1 \%$ of fund., modulation depths: $k_{5}=0.1$, and $k_{6,10,15,20,25}=0.01$, modulation frequency of the 6 th, 10th, 15 th, 20 th and 25 th harmonic $=1.7 \mathrm{~Hz} . f_{s}=3 \mathrm{kHz}, 15000$ samples, zero padding $=\times 2$.

Error on the modulation depth of the highest order harmonic

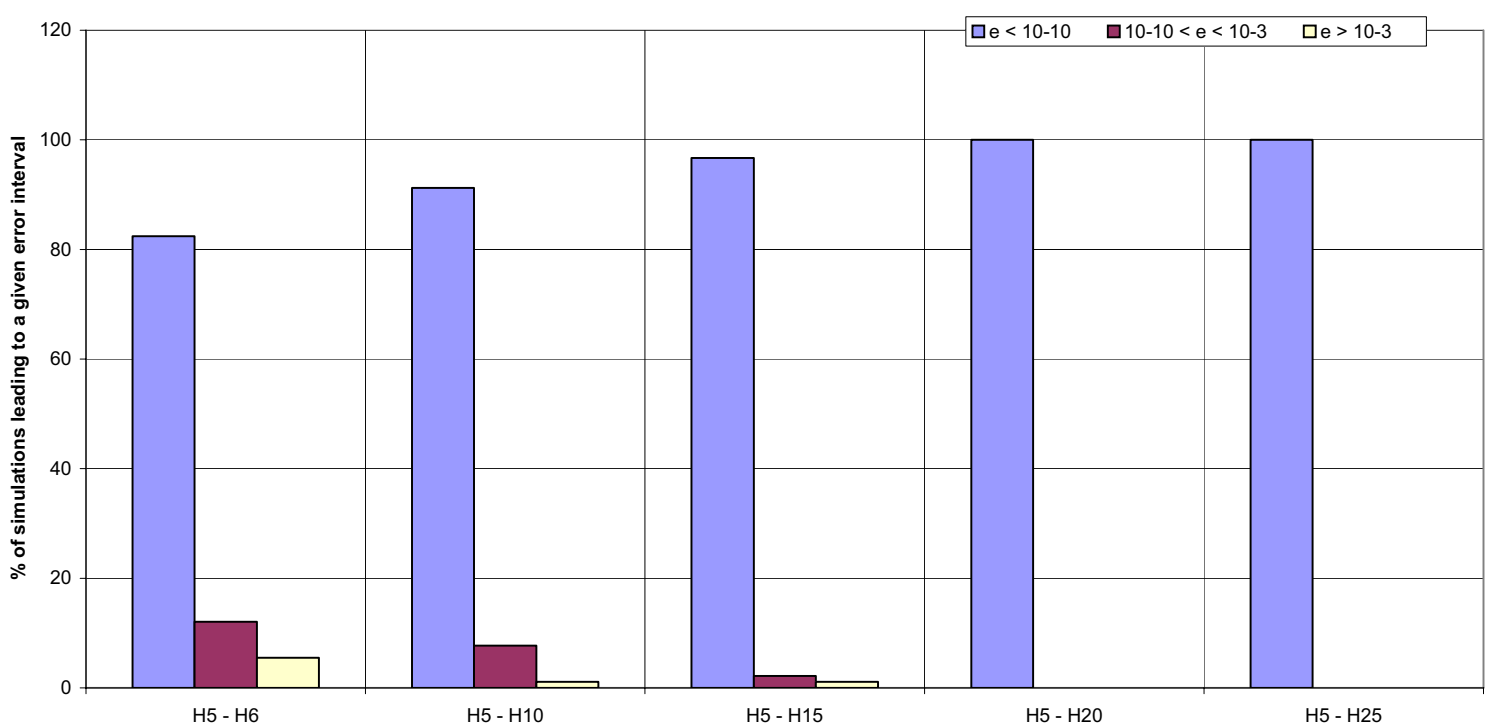

Fig. 15. (Color online) Influence of orders of the harmonic components on the error remaining after 10 iterations (histogram). $f_{0}=50 \mathrm{~Hz}$, magnitudes of harmonics: $10 \%$ and $1 \%$ of fund., modulation depths: 0.1 , and 0.01 , modulation frequency of the 6th, 10th, 15th, 20th and 25th harmonic $=1.7 \mathrm{~Hz} . f_{s}=3 \mathrm{kHz}, 15000$ samples, zero padding $=\times 2$.

fundamental component and the magnitudes and modulation depths of the harmonics without taking phases into account. In many cases, they led to calculation errors that are insignificant (a few parts in $10^{16}$ ) compared to uncertainty that should arise from AC voltage and current measurement instruments (a few parts in $10^{6}$ in the best cases). Some limitations of the method have been pointed out. Particularly when magnitudes and/or mod- ulation depths of harmonic components are significantly different, the resulting error can remain at a non acceptable level despite the iterative process. Nevertheless these errors could certainly be decreased by taking phases into account in the data processing. In our future work, we will implement these modifications and also the possibility to address other types of modulating waveforms. The possibility of analysing signals containing a larger number of 
Table 2. Summary of errors observed on the magnitude of the 6th order harmonic component.

\begin{tabular}{|c|c|c|}
\hline Mod. freq. $f_{m 6}$ & Equation: $f_{n(m, \alpha)}= \pm m f_{s} \pm f_{6} \pm(2 \alpha+1) f_{m 6}$ & Type of overlapping \\
\hline $1.6 \mathrm{~Hz}$ & $\begin{array}{l}f_{n(m, \alpha)}=m f_{s}-f_{6}+(2 \alpha+1) f_{m 6} \\
m=0 \\
-f_{6} \rightarrow(\text { negative part of the spectrum }) \\
\alpha=187\end{array}$ & Indirect covering \\
\hline $3.2 \mathrm{~Hz}$ & $\begin{array}{l}f_{n(m, \alpha)}=m f_{s}+f_{6}-(2 \alpha+1) f_{m 6} \\
m=2 \\
\alpha=937\end{array}$ & 2nd order aliasing \\
\hline $4.8 \mathrm{~Hz}$ & $\begin{array}{l}f_{n(m, \alpha)}=m f_{s}-f_{6}+(2 \alpha+1) f_{m 6} \\
m=0 \\
-f_{6} \rightarrow(\text { negative part of the spectrum }) \\
\alpha=62\end{array}$ & Indirect covering \\
\hline $6.4 \mathrm{~Hz}$ & $\begin{array}{l}f_{n(m, \alpha)}=m f_{s}-f_{6}-(2 \alpha+1) f_{m 6} \\
m=1 \\
\alpha=187\end{array}$ & 1st order aliasing \\
\hline $8 \mathrm{~Hz}$ & $\begin{array}{l}f_{n(m, \alpha)}=m f_{s}-f_{6}+(2 \alpha+1) f_{m 6} \\
m=0 \\
-f_{6} \rightarrow \text { (negative part of the spectrum) } \\
\alpha=37\end{array}$ & Indirect covering \\
\hline $9.6 \mathrm{~Hz}$ & $\begin{array}{l}f_{n(m, \alpha)}=m f_{s}+f_{6}-(2 \alpha+1) f_{m 6} \\
m=2 \\
\alpha=312\end{array}$ & 2nd order aliasing \\
\hline
\end{tabular}

harmonics will be considered. The last step of our work will be the application of this method to the real calibration of a programmable source of signals containing fluctuating harmonics that could then serve as a reference standard to establish the traceability of instruments used for on-site measurements.

Acknowledgements. This research, conducted within the EURAMET joint research project 'Power and Energy', has received partial support from the European Community's Seventh Framework Programme, ERANET Plus, under Grant Agreement No. 217257.

\section{Appendix A: Conditions on the modulation function}

First condition: the mean value of the modulation function should be equal to zero: this condition comes from the fact that the mean value of the magnitude of the $n$th order fluctuating harmonic (given by $\frac{1}{T_{\text {mod-n }}} \int_{t}^{t+T_{\text {mod-n }}} U_{n}\left[1+k_{n} \operatorname{Mod}_{n}\left(t, \phi_{\bmod -n}\right)\right] d t$, where $T_{\text {mod-n }}$ is the period of $\left.\operatorname{Mod}_{n}\left(t, \phi_{\bmod -n}\right)\right)$ should be equal to the magnitude $U_{n}$ of this harmonic in absence of modulation $\left(k_{n}=0\right)$ for any value of $n$.

Second condition: the minimum value of the modulation function should be equal to -1 : the second condition is necessary because the quantity $\left[1+k_{n} \operatorname{Mod}_{n}\left(t, \phi_{\text {mod-n }}\right)\right]$ has a physical meaning as being the "instantaneous" magnitude of this harmonic only if it remains positive or equal to 0 for any value of $t$ and of $k_{n}$ in the domain $0 \leqslant k_{n} \leqslant 1$.

\section{Appendix B: Detailed analysis of errors observed on the magnitude of the 6th order harmonic}

For this parameter, major errors occur when the modulation frequency of the 6 th order harmonic is equal to $1.6 \mathrm{~Hz}$, $3.2 \mathrm{~Hz}, 4.8 \mathrm{~Hz}, 6.4 \mathrm{~Hz}, 8 \mathrm{~Hz}$ and $9.6 \mathrm{~Hz}$ (see Fig. 5). These errors come from overlapping of the 6 th order harmonic by its own modulation components. Frequencies of modulation components relative to the $n$th order harmonic follow the equation $f_{n(m, \alpha)}= \pm m f_{s} \pm n f_{0} \pm(2 \alpha+1) f_{m h}$, where $f_{s}$ is the sampling frequency, $f_{0}$ the frequency of the fundamental component, $f_{m h}$ the modulation frequency of the $n$th order harmonic, $m$ and $\alpha$ integer numbers such as $m \geqslant 0$ and $\alpha \geqslant 0$. Overlapping of the 6 th order harmonic, which frequency $f_{6}$ is equal to $300 \mathrm{~Hz}$, by any modulation component occurs then when equation $f_{n(m, \alpha)}= \pm m f_{s} \pm f_{6} \pm(2 \alpha+1) f_{m h}=f_{6}$ is fulfilled. If $m \neq 0$ the overlapping is due to $m$ th order aliasing, otherwise to direct or indirect covering. Table 2 summarize values of $m$ and $\alpha$ that give rise to overlapping and indicates in each case the type of overlapping.

\section{Appendix C: Overlapping of the first modulation component by some other modulation component relative to the same harmonic, whatever the order of the fluctuating harmonic the modulation and the sampling frequencies}

Let us consider a signal consisting of a fundamental component at frequency $f_{0}$, and a $n$th order harmonic component fluctuating with a modulation frequency equal 
to $f_{m h}$ sampled at a sampling frequency $f_{s}$. Overlapping of the first modulation component by component arising from $m$ th order aliasing of the positive part of the spectrum occurs if the equation $m f_{s}+n f_{0}-(2 \alpha+1) f_{m h}=$ $n f_{0}+f_{m h}$ is fulfilled, that is equivalent to $m f_{s}=2(\alpha+$ 1) $f_{m h}$. As in the present simulation, all frequencies are defined with a $0.1 \mathrm{~Hz}$ resolution, it can be written $f_{s}=\frac{N_{s}}{10}$ and $f_{m h}=\frac{N_{m h}}{10}$ where $N_{s}$ and $N_{m h}$ are positive integer numbers. The previous equation is fulfilled if positive integer numbers $m$ and $\alpha$ such as $\frac{m}{\alpha+1}=\frac{2 N_{m h}}{N_{s}}$ can be found. One solution that always exists is $m=2 N_{m h}$ and $\alpha=N_{s}-1$.

\section{References}

1. V.K. Jain et al., High accuracy analog measurements via interpoled FFT, IEEE Trans. Instr. Meas. 28, 113-122 (1979)

2. T. Grandke, Interpolation algorithms for discrete fourier transform of weighted signals, IEEE Trans. Instr. Meas. 32, 350-355 (1983)

3. J. Wu, W. Zhao, New Precise Measurement Method of Power Harmonics Based on FFT, Proc. International Symp. Intel. Sig. Proc. And Comm. Syst. (2005), pp. 365368

4. L. Zhu et al., Extraction of periodic signal without external reference by time-domain average scanning, IEEE Trans. Instr. Meas. 55, 918-927 (2008)
5. I. Budovsky, G. Hammond, Precision measurement of power harmonics and flicker, IEEE Trans. Instr. Meas. 54, 483-487 (2005)

6. I. Budovsky, G. Hammond, Uncertainties in the measurement of power harmonics and flicker, CPEM 2004 Proc. (London, 2004), pp. 88-89

7. R. Arseneau, M. Sutherland, J. Zelle, A test system for calibrating flickermeters, IEEE Instrumentation and Measurement Technology Conference (Budapest, 2001), pp. $408-411$

8. P.S. Wrigth, P. Clarkson, Methods for the Calibration of Flickermeters, NCSL International (Washington DC, 2001)

9. P. Espel, Comparison of three accurate methods for flicker measurements, Metrologia 47, 287-294 (2010)

10. R. Arseneau, M.E. Sutherland, J.J. Zelle, S. Svensson, Comparison on nonsinusoidal calibration systems at NRC Canada and SP Sweden, IEEE Trans. Instr. Meas. 50, 275$277(2001)$

11. P.S. Wrigth, Polynomial decomposition of smoothly fluctuating harmonics applied to the calibration of harmonic analysers, IEEE Trans. Instr. Meas. 54, 492-495 (2005)

12. P.S. Wrigth, A method for the calibration of harmonic analysers using signals containing fluctuating harmonics in support of IEC61000-3-2, IEE Proc.-Sci. Meas. Technol. 152, 103-109 (2005)

13. P. Clarkson, P. Wright, A wavelet-based method of measuring fluctuating harmonics for determining the filter time constant of IEC standard harmonic analysers, IEEE Trans. Instr. Meas. 54, 488-491 (2005) 